\title{
The use of polyurethane foam waste resulting from the technological process of insulation of refrigeration enclosures in obtaining constructions panels with sound-absorbing and thermally insulating properties
}

\author{
Laurentiu Marin ${ }^{1 *}$ \\ ${ }^{1}$ National Institute for Research and Development in Chemistry and Petrochemistry - ICECHIM \\ Bucharest, Splaiul Independentei, no.202, sector 6, 060021, Bucharest, Romania
}

\begin{abstract}
Refrigerated enclosures - refrigerators, refrigerated units, vans for transporting perishable goods are thermally insulated with polyurethane foam. The insulation process involves the pressure injection of the component reactants into the spaces to be insulated. Following the polyaddition reaction, the foaming-expansion process takes place with an increase in volume. Finally, a spongy polymeric mass is obtained, which filled the spaces where insulation had to be carried out and which also generated an excess of material which is subsequently removed by mechanical means. Excess material results from the initial dosage of the insulation material and must exist to ensure that the spaces to be insulated are completely filled. Polyurethane foam waste is a real problem for several reasons. With a large volume, it takes up a lot of storage space with unpleasant consequences, polyurethane foam is a slightly non-degradable reactive material that persists in the environment. The combustion of polyurethane foam waste is not an option to be adopted because the combustion, in addition to $\mathrm{CO}_{2}$, also results a series of particularly toxic substances: isocyanates, phosgene, cyan.
\end{abstract}

\section{Introduction}

Modern construction technologies (especially in the case of office buildings) have abandoned the old system of partitioning through walls, with the creation of fixed spaces, well determined, non-removable with or without connection between them and have adopted the process of partitioning through removable walls that are easy to assemble and disassemble just as easily. They have the possibility to be painted, painted, and on them can be attached different ornamental objects or technologically useful objects.

The use of removable panels has the advantage that the topography of the place can be easy modified when the situation requires it, without affecting the resistance structure of the building. Removable panels are $20-40 \mathrm{~mm}$ thick plasterboard. They have the advantage that

\footnotetext{
* Corresponding author: andree marr@yahoo.co.uk
} 
they can be processed by cutting or drilling and can be painted just like an ordinary wall. The disadvantages of these panels are the friability and the reduced capacity of sound absorption. in buildings where activities in various fields are carried out, silence is a mandatory attribute. This disadvantage is removed by the product which is the subject of this patent application, a complex structure of layered panel which consists of two upper layers of gypsum that are connected to each other by a granulated polyurethane foam filling, obtained by grinding polyurethane foam waste resulting from the technological processes of insulation of refrigeration enclosures. Polyurethane foam grinding is recovered from waste. The realization of this laminate does not alter at all the physical properties of the facade of the gypsum panels, instead it determines an increased capacity of vibration absorption and the resistance of the panel to accidental breakage improvement. The inner layer sound-absorbing does not alter the machinability of the panel by various methods (drilling cutting) nor the possibility of its subsequent painting. The inner layer has the role of sound absorption - especially of those with low frequency, and also being elastic and adherent to the gypsum board to reduce its friability reducing the risks of accidental breakage of gypsum boards during transport or installation. The sound-absorbing material is ground polyurethane foam, recovered from the technological waste resulting from the processes of obtaining refrigerators, insulating metal panels, pre-insulated pipes, waste difficult to destroy by ecological processes.

\section{The achievement of sound-absorbing panels of plasterboard}

The panel with sound-absorbing properties usable in constructions is a complex structure made up of plasterboard faces that have a sound-absorbing active component in the middle, obtained from polyurethane foam waste. The two components are bonded together with a polyurethane binder. The polyurethane binder is used because it is compatible with both components of the sound-absorbing structure [1].

\subsection{Preparation of sound-absorbing material}

Polyurethane foam waste that can be collected by conventional recoverable waste reporting processes is separated from impurities - especially from metallic impurities. After separating the impurities, the foam waste is ground in a grinding device with rotating knives. The grinding process is maintained until the polyurethane foam waste reaches an average size of $10-15 \mathrm{~mm}$. The size of the polyurethane foam granules is checked visually after stopping the grinding device and placing the foam granules in the tank of the device. It is to be avoided a more accentuated grinding of the polyurethane foam waste because after the mixing process with the binder that will bind them together and at the same time will stick them to the inside of the gypsum panels because after binding and subsequently pressing between the two panels would create a compact mass of material that would not have the required sound-absorbing properties as seen in Figure 1. In general, the crushing process lasts 30 - 40 seconds considering the high friability of the foam waste as well as the technological indication to avoid the excessive crushing of the polyurethane foam waste. Blade rotation speed of the crushing device was 130-150 revolutions / minute. 


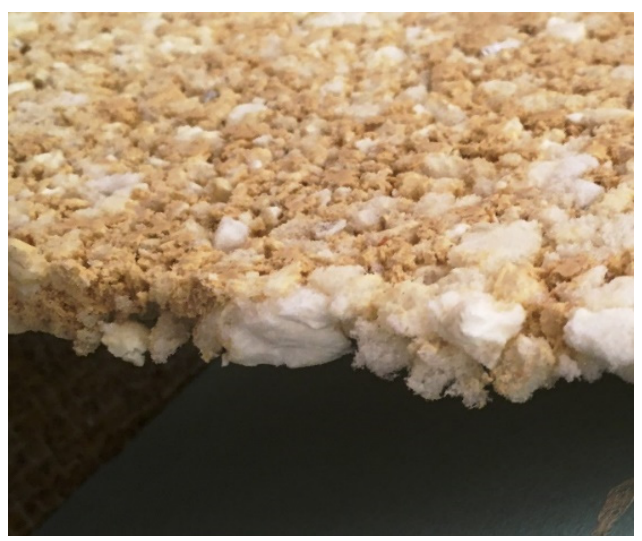

Fig. 1. Granular material obtained by grinding polyurethane foam waste.

After grinding, the crushed material is removed from the tank of the device, it is separated from the foam dust resulting from the grinding by sieving and stored until further proces-sing.

\subsection{Obtaining the polyurethane binder.}

The polyurethane binder is of two-component type. The component A contains the polyetherpolyol PETOL 36 Type currently in production at Oltchim Ramnicu Valcea [2], and all the elements of the recipe, and component $\mathrm{B}$ is the crosslinking agent - an aromatic diisxocyanate 4,4 'diisocyanate diphenyl methane.

\subsubsection{Polyether polyol synthesis reaction}

The polymer matrix is constructed starting from a polyether polyol type PETOL 36 currently in production at OLTCHIM. The synthesis of this polyether starts from a polyaddition reaction of an -OH group to an epoxy group. Being a polyether 3 the number 1 reactant is glycerin. Reactant number 2 is propylene oxide. The stoichiometry of the reaction is chosen so that at the end to obtain a polyether polyol with molecular mass 4800 5000 UAM.

\subsubsection{Modifying the composition of the polyether polyol and obtaining component A}

PETOL-type polyol is introduced into a reaction vessel together with a resin solution in an organic solvent. The stirring is leave for 30 minutes to homogenize the components. The resin is needed to ensure subsequent stickiness. Then the UV stabilizer, the inorganic filler and the reaction catalyst are added. The reaction catalyst is added in a proportion that can vary from case to case. The proportion of catalyst determines the processing time of the composition (pot-life) [3]. At the end a quantity of crosslinker is added but in a substoichiometric proportion in order not to crosslink the composition. After homogenizing the components of the recipe, it is transferred from the reaction vessel to a metal storage container. Storage can be done indefinitely as long as the necessary crosslinker is not added. 


\subsubsection{Crosslinker component $B$}

The crosslinker is mandatory a diisocyanate to determine a polyaddition reaction with the formation of a polyurethane macromolecule [4]. It was chosen 4,4 'diisocyanate diphenyl methane because it is less volatile than other diisocyanates so that it does not create health problems for the operators and does not require respiratory protection equipment for them [5-8]. in order to achieve the crosslinking of the composition, in component A a percentage of $15-20 \%$ gravimetric parts is added crosslinking agent $[9,10]$.

\subsubsection{Preparation of polyurethane foam waste granules}

The collected polyurethane foam waste is in the form of lumps of irregular shapes and sizes also varied. After collection, impurities and dirt are removed so that only polyurethane foam remains. The foam waste is introduced into a mill with knives that grinds it to 8-15 $\mathrm{mm}$ grain sizes. Polyurethane foam waste granules are stored after grinding in containers or bags until use. Their storage can also be done indefinitely.

\subsubsection{Obtaining sound-absorbing panels}

Obtaining sound-absorbing panels involves a series of preliminary operations. These are followings: preparation of the adhesive for binding the foam granules, mixing the foam granules with the adhesive, mixing the foam granules with the adhesive and homogenizing the mixture for wetting the foam granules with the adhesive, making foam waste boards, preparation of gypsum boards by wetting them with adhesive on the faces that come in contact with the foam waste boards, attaching below and above the polyurethane foam waste board the gypsum boards with the faces moistened with adhesive on the foam waste board, pressing the assembly for 30 minutes.

\subsubsection{Preparation of the binder}

To achieve the technological properties for which it was developed, the polyurethane adhesive must be prepared as follows: A quantity of component $\mathrm{A}$ is weighed in a work container of suitable size. In component A under continuous stirring, a quantity of crosslinker is added in a proportion of $15-20 \%$ gravimetric parts. The stirring is kept until homogenization. From this moment the adhesive is suitable for use. the use of the adhesive must be completed before the expiration of the crosslinking time (pot life). The adhesive recipe was designed in such a way as to give it a pot life of about 30 minutes [7].

\subsubsection{Obtaining the sound-absorbing plate from foam waste}

It weighs an adequate amount of polyurethane foam waste granules. This amount is introduced into a mixer with sigma arms. Stirring is started and a three times larger amount of crosslinkable polyurethane adhesive is introduced under stirring. This proportion is necessary for technological reasons. the density of the foam granules is much lower than the density of the adhesive and it is mandatory for the entire amount of granules to be moistened in order to bind them. Mixing the polyurethane foam waste granules in the sigma arm mixer is maintained for about 12-15 minutes until all the foam waste granules are 
moistened with the crosslinkable adhesive. The mixing must not exceed this time because the adhesive can crosslink in the tank and can no longer ensure the necessary binding later. After the mixing / wetting operation of the foam granules, the mixture is removed from the mixer tank and placed in a mold that ensures a flat shape later. After filling the mold, it closes and a light pressing is applied. the mold is made of polyethylene so that the adhesive used to bind the granules does not adhere to its walls and at the same time after binding the resulting plate can be removed from the mold. After removing from the mold the plate that ensures the sound-absorbing properties is shown in Figure 2.

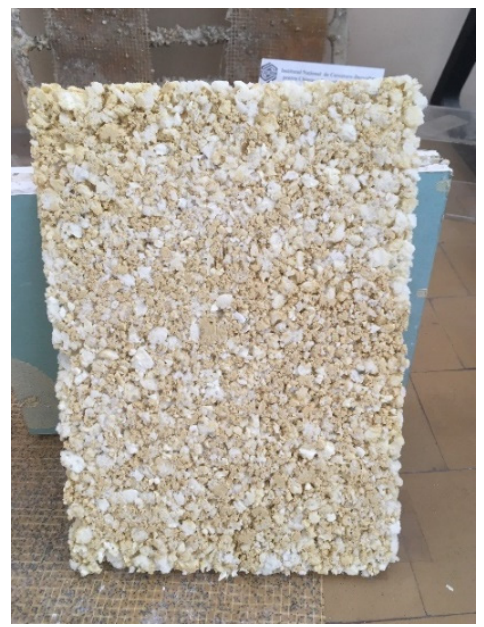

Fig. 2. Foam waste soundproof plate.

\subsubsection{Obtaining gypsum sound-absorbing panels}

Two gypsum boards are moistened on each side with crosslinked adhesive prepared according to point 2.2.5.1. The adhesive can be deposited by brushing, rolling or using a compressed air device for painting. The adhesive is deposited in an amount of about 30-40 $\mathrm{g} / \mathrm{m}$.p. With the wet adhesive on the adhesive face of the panel number 1, place the foam waste plate obtained according to pt. 2.2.5.2.
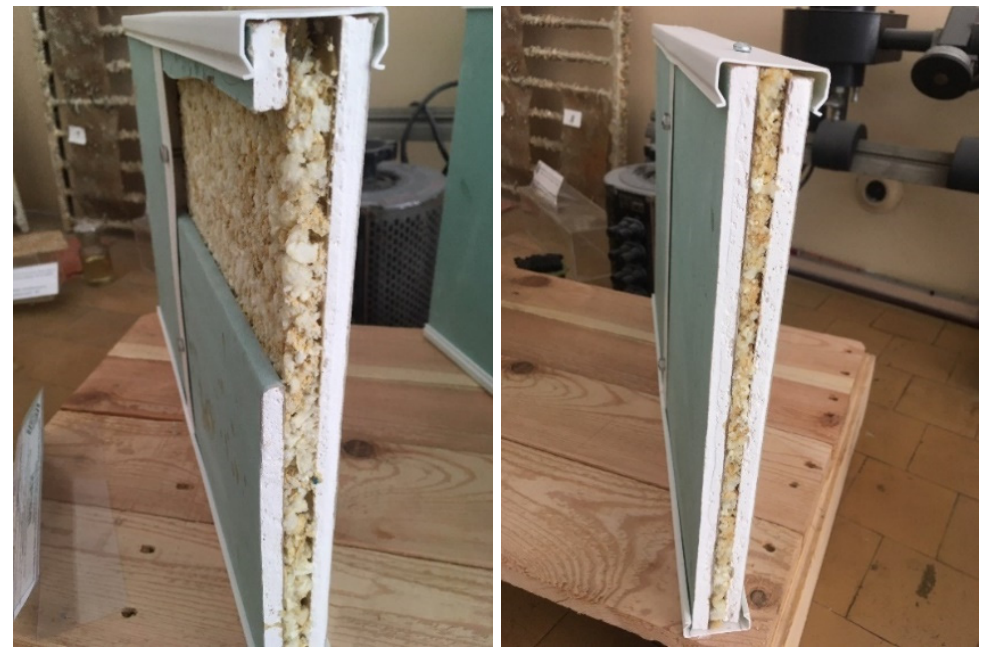

Fig.3. Foam waste soundproof plate. 
Above the sound-absorbing layer is placed the gypsum board number 2 with the adhesive face on the sound-absorbing layer of foam waste. the assembly thus obtained is introduced in a press where a slight overpressure is applied. The complex is kept in the press for about 20-25 minutes. after removing from the press the gypsum board can be used. The finished product is shown in Figure 3.

The finished product has superior properties to the initial gypsum panels not only in terms of noise absorption capacity but also their reduced friability. The finished products can be installed and used in the same way as the initial gypsum board.

\section{Conclusions}

The researchers identified a process for the superior use of this waste - which also materialized in a patent application $A$ 2020-00057 / 07.02.2020 Sound-absorbing structure from polyurethane waste and it result from the research contract 51PCCDI / 2018. Thus, the polyurethane foam waste is crushed to a granulation of $8-10 \mathrm{~mm}$. The material thus obtained is mixed with a polyurethane binder in a proportion of 1 granulated waste 2 polyurethane binder. This mixture is placed evenly on the surface of a construction panel and another panel is placed on top. Finally, a layer is obtained which has in the middle the granular structure made of the mixture of crushed polyurethane binder waste. The polyurethane binder also achieves the adhesion of the structure to the wall of the panel.

\section{References}

1. O. Gunter, Polyurethane Handbook (New York: Macmillen Publishing Co. Inc. 1985), ISBN $\underline{0-}$ 02-948920-2

2. Catalog prezentare produse OLTCHIM S.A., p. 12-16 (2012)

3. Synthesis of Organometallic Compounds: A Practical Guide Sanshiro Komiya Ed. 1997

4. A.K. Smith, R.J. Goddard, E.J.L. Paulsen, MDI-based polyurethane prepolymer with low monomeric MDI content, US patent 6884904, (2005)

5. Health and Safety Executive, Guidance Note (EH16) Isocyanates: Toxic Hazards andPrecautions (1984)

6. Material Safety Data Sheets: Poly-Foam Comp. "A" \& "B"; SWD 850 "A" \& "B"; Froth-Pak NIOSH Alert (No.96-111), Preventing Asthma and Death from Diisocyanate Exposure (1996)

7. L Martin, Nanocompozite polimerice pentru acoperiri podele institutii publice, PN. 09.09.04.13 F.1, p. 37

8. P. Werle, M. Morawietz, S. Lundmark, K. Sörensen, E. Karvinen, J. Lehtonen, Alcohols, Polyhydric, Ullmann's Encyclopedia of Industrial Chemistry, (Wiley-VCH, Weinheim, 2008) doi:10.1002/14356007.a01_305.pub2

9. J. March, Advanced Organic Chemistry: Reactions, Mechanisms, and Structure (ed. 3), (New York: Wiley, 2010), ISBN 0-471-85472-7

10. C. Six, F. Richter, Isocyanates, Organic, in Ulmann's Encyclopedia of Industrial Chemistry, (Wiley-VCH, Weinheim, 2005) doi:10.1002/14356007.a14_611 